\title{
Ejemplares singulares de Eucalyptus cladocalyx F. Muell. testigos del arboreto histórico de Sierra Cabello (San Bartolomé de la Torre, Huelva)
}

\section{Singular specimens of Eucalyptus cladocalyx F. Muell. witnesses of the historic arboretum of Sierra Cabello (San Bartolomé de la Torre, Huelva)}

\author{
Butler Sierra I. ${ }^{*}$, Monteagudo Sánchez de Movellán F. J. ${ }^{1}$ \\ ${ }^{1}$ Universidad de Huelva, Departamento de Ciencias Agroforestales, \\ Campus Universitario La Rábida, 21819 Palos de la Frontera Huelva
}

*Autor para correspondencia: butler@dcaf.uhu.es

\section{Resumen}

Los eucaliptos se encuentran presentes en la provincia de Huelva desde mediados del siglo XIX en plantaciones de numerosas especies promovidas por empresas y propietarios particulares. Una de las mejor documentadas, gracias a los trabajos de Manuel Martín Bolaños, es la de Sierra Cabello (San Bartolomé de la Torre) creada por José Duclós a partir de 1918. La citada finca ha sufrido grandes transformaciones a lo largo del tiempo, pero del antiguo arboreto de eucaliptos aún permanecen en pie algunos ejemplares de grandes dimensiones destacando los de Eucalyptus cladocalyx. Mediante esta comunicación se propone la inclusión de Sierra Cabello y sus ejemplares notables de E. cladocalyx en el Inventario de Árboles y Arboledas Singulares de Andalucía con objeto de fomentar su conocimiento y protección.

\section{Summary}

Eucalypts are present in the province of Huelva since the mid-nineteenth century in plantations of numerous species promoted by companies and private owners. One of the best documented, thanks to the work of Manuel Martín Bolaños, is that of Sierra Cabello (San Bartolomé de la Torre) created by José Duclós from 1918. The aforementioned farm has undergone great transformations over time, but from the ancient eucalyptus arboretum some large specimens still stand out, highlighting those of Eucalyptus cladocalyx. This communication proposes the inclusion of Sierra Cabello and its notable specimens of E. cladocalyx in the Inventory of Singular Trees of Andalusia in order to promote their knowledge and protection.

Palabras clave: Biogeografía, conservación, Duclós, Martín Bolaños

Keywords: Biogeography, conservation, Duclós, Martín Bolaños 


\section{Introducción y objetivos}

Desde que en 1974 el Instituto para la Conservación de la Naturaleza-ICONA promoviera la creación de un "Inventario de árboles monumentales de España" comienza a vislumbrarse la protección de individuos con características señeras como herramienta para su conservación. Aunque el trabajo no llegó a completarse, durante las décadas siguientes se desarrollaron nuevas iniciativas a nivel autonómico y local, formándose finalmente el actual marco normativo relativo a los denominados "árboles singulares" (Rigueiro Rodríguez, doc. ined. 2016).

La normativa que regula el "Catálogo Andaluz de Árboles y Arboledas Singulares" (BOJA 2003 y 2012), de forma análoga a la normativa de otras comunidades autónomas, establece que cualquier ciudadano u organización puede solicitar el inicio de un procedimiento de declaración a la Dirección General competente en materia de conservación de la flora y la fauna silvestres de la Consejería de Medio Ambiente y Ordenación del Territorio. En la solicitud se ha de justificar la "singularidad" de ejemplares arbóreos individuales o de agrupaciones que destaquen por: sus características morfológicas o dendrométricas; su longevidad o belleza; la rareza de la especie a la que pertenezcan, relativa tanto al número de individuos como al área de distribución, o por sus particularidades científicas; su ubicación en emplazamientos o en ambientes bioclimáticos o biogeográficos poco habituales; su relación con acontecimientos históricos, culturales o populares relevantes; o su valor paisajístico. Prácticamente todas estas circunstancias concurren en los vestigios de la antigua plantación de eucaliptos de Sierra Cabello, localizada al sur de la población de San Bartolomé de la Torre en el Andévalo onubense.

El objetivo de esta comunicación es describir el antiguo arboreto de Sierra Cabello y sus características más destacadas y proponer la inclusión de Sierra Cabello y sus ejemplares notables de Eucalyptus cladocalyx F. Muell. en el Inventario de Árboles y Arboledas Singulares de Andalucía con objeto de fomentar su conocimiento y protección.

\section{Material y métodos}

Gracias al interés de Manuel Martín Bolaños por la plantación de Sierra Cabello se dispone de una inusual documentación para su estudio. Parte de ella se encuentra publicada describiendo sus aspectos botánicos y selvícolas (Martín Bolaños 1928, 1946 y 1955), aunque ha sido posible completarla con:

- La correspondencia entre Martín Bolaños, los propietarios de la finca, José Duclós (1942-1947) y su hijo Carlos Duclós (1949-1951), y el ingeniero del Patrimonio Forestal del Estado Gaspar de la Lama (1951).

- La colección Eucalyptus del Herbario MAIA del Centro de Investigación Forestal del Instituto Nacional de Investigación y Tecnología Agraria y Alimentaria-CIFOR INIA.

- La Fototeca Histórica Martín Bolaños de la Universidad de Huelva. 
— Muestras herborizadas en 1946 en Sierra Cabello conservadas en MAIA sin identificar y catalogar y fotografías tomadas por Martín Bolaños archivadas en la Unidad Docente de Botánica de la Escuela Técnica Superior de Ingenieros de Montes de la Universidad Politécnica de Madrid- ETSIM UPM (Butler Sierra, 2016).

Tras el análisis de la documentación, se ha realizado un estudio de la flora, vegetación y singularidad en la zona forestal actual de la finca, así como la obtención de parámetros dendrométricos destacados de E. cladocalyx mediante Blume-Leiss y cinta métrica.

\section{Resultados y Discusión}

Situada al SO del municipio onubense de San Bartolomé de la Torre sobre un llano arenoso, Sierra Cabello se eleva en dirección SO-NE constituida por dos mesetas de cota máxima 199 m (Fig. 1) construidas con materiales de depósito cuaternarios, principalmente conglomerados, arenas y gravas (Instituto Geológico y Minero de España, 1983). El clima suave con marcada sequía estival (Tab. 1) mitigada por la presencia de agua a escasa profundidad, aproximadamente a $3 \mathrm{~m}$ en verano (Martín Bolaños, 1946), y por la interceptación de la humedad de los vientos atlánticos en esta primera elevación situada a escasos kilómetros de la costa, hicieron de Sierra Cabello, a pesar de la escasa fertilidad de su suelo, una finca adecuada para la explotación de eucaliptos por parte de su propietario José Duclós desde 1918 hasta su muerte a finales de los años 40 del siglo XX.

Tabla 1. Datos climáticos de la estación meteorológica de San Bartolomé de la Torre (Huelva). Periodo de datos: térmicos 1970-1989, pluviométricos 1946-1989.

\begin{tabular}{ll}
\hline Tm anual & $18.6^{\circ} \mathrm{C}$ \\
\hline P anual & $636 \mathrm{~mm}$ \\
\hline Tm mes más cálido & $27.2^{\circ} \mathrm{C}$ (julio) \\
\hline Tm mes más frío & $11.2^{\circ} \mathrm{C}$ (enero) \\
\hline P mes más húmedo & $95 \mathrm{~mm}$ (diciembre) \\
\hline P mes más seco & $2 \mathrm{~mm}$ (julio) \\
\hline Bioclima & Mediterráneo Pluviestacional-Oceánico \\
\hline Termotipo & Termomediterráneo Inferior \\
\hline Ombrotipo & Seco Superior \\
\hline
\end{tabular}

Fuente: Rivas Martínez, 2007

El Ingeniero de Montes Manuel Martín Bolaños, natural de Huelva y amigo de Duclós, se sintió interesado desde joven por los "árboles australianos", constituyéndose con el tiempo en un reconocido especialista en esta materia a nivel nacional e internacional. Gracias a esta relación personal conoció Sierra Cabello y se enrique- 


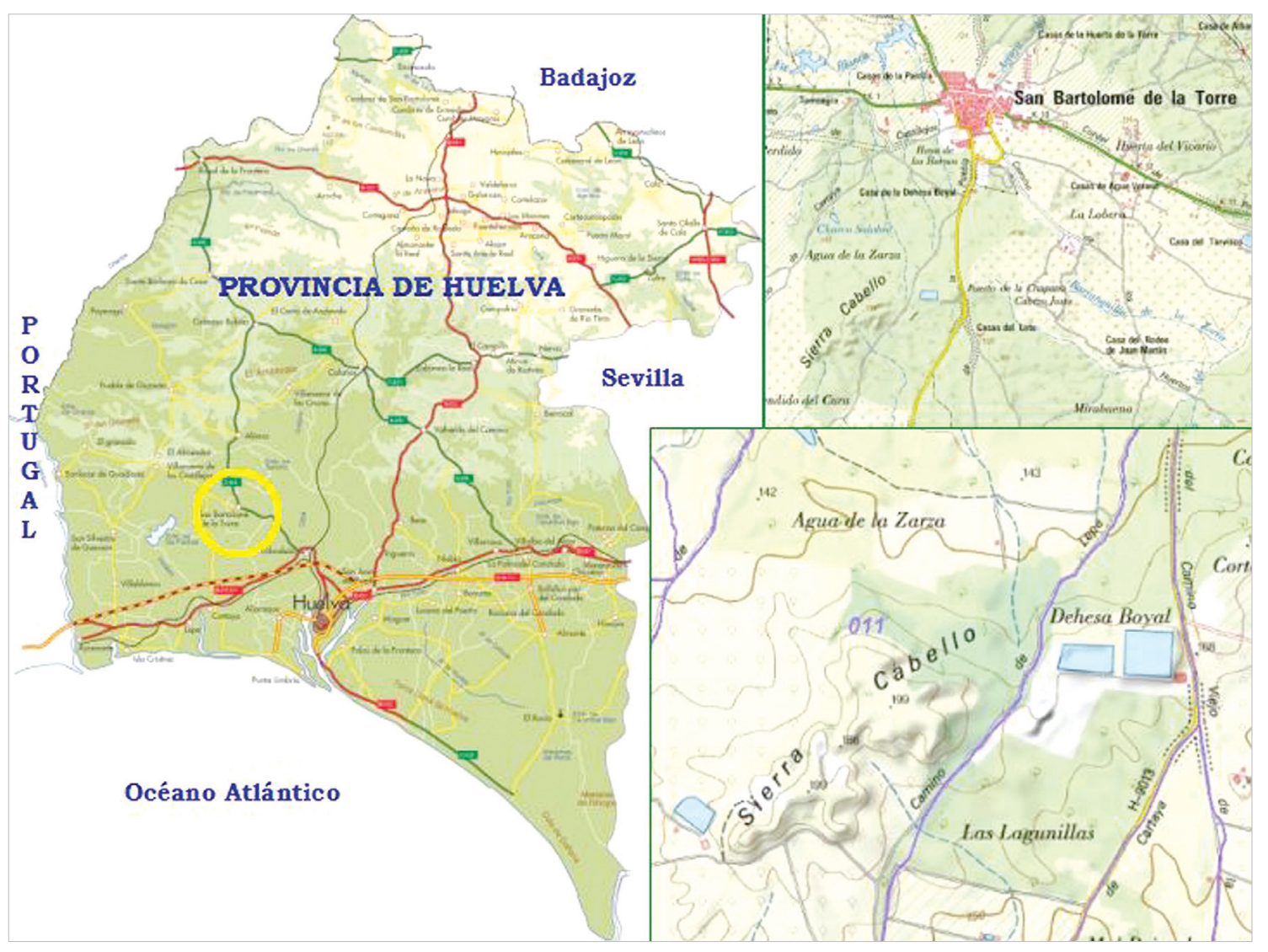

Figura 1. Mapas de localización de Sierra Cabello (San Bartolomé de la Torre, Huelva).

ció con los conocimientos empíricos de su propietario y con los extraídos de la biblioteca especializada que este había formado con el paso de los años. Martín Bolaños (1928 y 1946) documentó mediante publicaciones ilustradas con fotografías comentadas esta plantación en dos momentos distanciados por un periodo de casi 20 años, siendo testigo del incremento del número de especies ensayadas gracias a las semillas enviadas desde Australia por un amigo inglés al que Duclós, en pago, remitía garbanzos. Además, en 1931, 1946 y 1951 tomó para su estudio muestras de diferentes especies de eucaliptos junto a Duclós y por encargo suyo, Eusebio Roberto Alonso recolectó muestras de especies del rico matorral propio de este territorio (Tabs. 2 y 3), que pasaron a engrosar el herbario del Instituto Forestal de Investigaciones y Experiencias-IFIE, actual Herbario MAIA del CIFOR-INIA.

La cubierta vegetal de la finca anterior a la plantación era un matorral muy diverso destinado a alimentar al ganado cabrío, con presencia puntual de pinos (Pinus pinea L.), alcornoques (Quercus suber L.) e higueras (Ficus carica L.). Su transformación en eucaliptal comenzó con la plantación entre 1918 y 1924 de 100 ha con $E$. globulus Lab. y E. camaldulensis Dehnh. ensayando en años posteriores hasta un total de 45 especies de eucaliptos, destinándose a la obtención de madera y esencia (Fig. 2, Tab. 2) (Martín Bolaños 1928 y 1946). Duclós y Martín Bolaños proyectaron entre 1942 y 1946 organizar de cara al futuro el arboreto conseguido identificando las 
diferentes especies con cartelería de hierro lacada en blanco y negro, trabajo que acometería el IFIE sin gastos para el propietario. Esto último era importante ya que Duclós no debía pasar por una situación económica desahogada, lo que llevó a que en 1942 Martín Bolaños le propusiera la compra de la biblioteca por parte del IFIE y que años más tarde, en 1951, éste y Gaspar de la Lama Gutiérrez transmitieran a sus hijos Carlos y Francisco Duclós una oferta del Patrimonio Forestal del Estado de 2 millones de pesetas para la adquisición de la finca, la biblioteca, las notas de las experiencias realizadas y la casa que poseían en San Bartolomé de la Torre, operación que no terminó de fraguar por el interés de los herederos en continuar la obra de su padre.

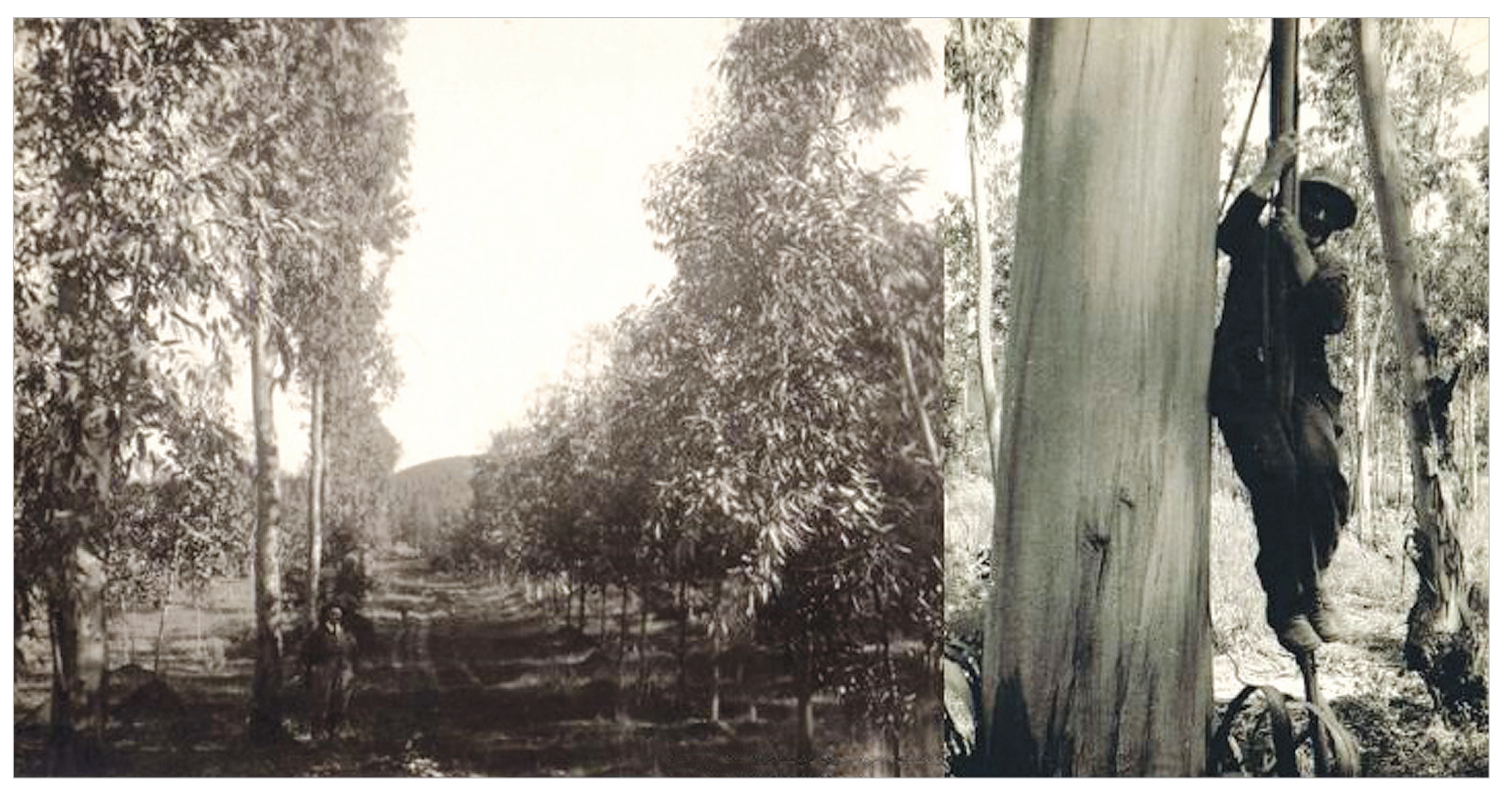

Figura 2. Fotografías tomadas por Manuel Martín Bolaños en Sierra Cabello. Izquierda: José Duclós junto a un ejemplar de Eucalyptus camaldulensis de 8 años de edad y $18 \mathrm{~m}$ de altura; a su derecha E. resinifera de 3 años y 6 m (enero de 1928). Derecha: eucalipto de notable diámetro (mayo 1951).

Desde entonces, mucho ha cambiado el paisaje. Gran parte de la finca se ha destinado desde los años 90 al cultivo intensivo de cítricos y se han realizado extracciones de áridos que han dejado una huella evidente (Fig. 3). La vegetación actual de Sierra Cabello muestra entre los restos del antiguo eucaliptal rodales y pies sueltos de alcornoque mezclados puntualmente con encina (Q. ilex subsp. ballota (Desf.) Samp.) y madroño (Arbutus unedo L.), que conectan con el alcornocal de la Dehesa Boyal de San Bartolomé de la Torre. Sobre el llano arenoso se extiende un denso pinar de pino piñonero y matorral samófilo formado por jaguarzos (Halimium halimifolium Willk., H.calycinum (L.) K. Koch, Cistus libanotis L.), bayón (Osyris lanceolata Hochst. \& Steud.), mirto (Myrtus communis L.), cantueso (Lavandula pedunculata (Mill.) Cav.), romero (Rosmarinus officinalis L.) etc., con presencia notable del endemismo del SW peninsular Armeria velutina Welw. ex Boiss. \& Reut. En las laderas acarcavadas se instala un brezal-aulagar con madroño muy característico de Erica 
Tabla 2. Relación de las 45 especies plantadas en el arboreto de Sierra Cabello por D. José Duclós y fecha en la que está documentada su presencia. *: 31 especies de correcta identificación constatada por la bibliografía consultada o por los pliegos del Herbario MAIA.

\begin{tabular}{|c|c|c|c|}
\hline Periodo & \multicolumn{3}{|c|}{ Especies del género Eucalyptus } \\
\hline 1918 & E. camaldulensis* & E. globulus* & \\
\hline \multirow[t]{3}{*}{ 1919-1924 } & E. botryoides & E. cornuta & E. resinifera \\
\hline & E. cladocalyx & E. maideni & E. robusta \\
\hline & E. colossea & E. obcordata & E. tereticornis \\
\hline \multirow{6}{*}{$\begin{array}{l}1946 \\
\text { no constatada identificación } \\
\text { por Martín Bolaños }\end{array}$} & E. acmenioides & E. hemilampra & E. pulverulenta \\
\hline & E. angustissima & E. leucoxylon & E. sideroxylon \\
\hline & E. calophylla & E. lehmannii & E. tereticornis \\
\hline & E. capitellata & E. longofolia & E. tetraptera \\
\hline & E. goniocalyx & E. oblicua & E. viminalis \\
\hline & E. gunnii & E. polyanthemos & \\
\hline \multirow{7}{*}{$\begin{array}{l}1946 \\
\text { constatada identificación } \\
\text { por Martín Bolaños }\end{array}$} & E. amplifolia* & E. diversicolor* & E. occidentalis* \\
\hline & E. botryoides* & E. eugenioides* & E. platypus* \\
\hline & E. camaldulensis & E. globulus & E. resinifera* \\
\hline & E. cinerea* & E. gomphocornuta* & E. robusta* \\
\hline & E. citriodora* & E. kirtoniana* & E. rudis* \\
\hline & E. cladocalyx* & E. maculata* & E. saligna* \\
\hline & E. cornuta* & E. maidenii ${ }^{*}$ & E. trabutii* \\
\hline \multirow{7}{*}{$\begin{array}{l}\text { Herbario MAIA } \\
\text { pliegos 1931-1951 }\end{array}$} & E. amplifolia & E. maculata & E. scabra* \\
\hline & E. botryoides & E. paniculata * & E. sideroxylon* \\
\hline & E. camaldulensis & E. polyanthemos* & E. smithii* \\
\hline & E. capitellata* & E. pulverulenta* & E. tenuissima ?* \\
\hline & E. citriodora & E. resinifera & E. trabutii \\
\hline & E. cladocalyx & E. robusta & E. umbellata* \\
\hline & E. kirtoniana & E. saligna & E. viminalis* \\
\hline
\end{tabular}

Fuente: Martín Bolaños, 1928 y 1946; Herbario MAIA Colección de eucaliptos, CIFOR-INIA

Tabla 3. Relación de las especies recolectadas en Sierra Cabello, en junio de 1946, por Eusebio Roberto Alonso para los trabajos de flora y vegetación de Martín Bolaños.

\begin{tabular}{|c|c|c|}
\hline Familia & Especie & Nombre vernáculo asignado \\
\hline \multirow[t]{3}{*}{ Cistaceae } & Cistus libanotis & \multirow{4}{*}{ Jaguarzo } \\
\hline & Halimium halimifolium & \\
\hline & Tuberaria lignosa & \\
\hline Cruciferae & Iberis ciliata subsp. welwitschii & \\
\hline Cupressaceae & Juniperus navicularis & Aulaga \\
\hline \multirow[t]{3}{*}{ Ericaceae } & Arbutus unedo & Madroña \\
\hline & Erica arborea & Brezo \\
\hline & Erica ciliaris & Brezo \\
\hline Fagaceae & Quercus $x$ morisii & Mesto \\
\hline \multirow[t]{2}{*}{ Labiatae } & Lavandula pedunculata & \\
\hline & Rosmarinus officinalis & Romero \\
\hline \multirow[t]{2}{*}{ Leguminosae } & Stauracanthus boivinii & Aulaga \\
\hline & Ulex minor & Aulaga \\
\hline Santalaceae & Osyris lanceolata & \\
\hline
\end{tabular}

Fuente: Butler Sierra, 2016 
australis L., E. umbellata L. y los endemismos del SW peninsular Stauracanthus boivinii (Webb) Samp. y Ulex argenteus subsp. sericeus (Cout.) Rothm., en el que debió integrarse el enebro Juniperus navicularis Gand., única cita existente para el Andévalo onubense, desaparecido por el cambio de uso acaecido. Sobre este conjunto destacan las copas aisladas de algunos grandes eucaliptos, los rostrata o rojos $E$. camaldulensis y blancos E. globulus, habituales en la Baja Andalucía, y los más exóticos y peculiares eucaliptos del azúcar E. cladocalyx.

E. cladocalyx, es originario de los montes esclerófilos secos del sur de Australia y del NO de la Isla de los Canguros, donde habita en áreas de precipitaciones entre 450 y $625 \mathrm{~mm}$ concentradas en invierno sobre suelos esqueléticos de escasa fertilidad procedentes de cuarcitas y rocas sedimentarias (Martín Bolaños, 1955), condiciones mesológicas similares a las de Sierra Cabello. A pesar de ser una especie muy difundida por repoblación en Australia fuera de su área de distribución, en España ha tenido un uso muy limitado circunscrito a la Baja Andalucía, en estaciones libres de heladas a las que es muy sensible. Hoy en día en Huelva es una especie rara, estando representada en los arboretos de El Villarejo (Almonte), El Villar (Bonares), La Garnacha (Cortegana), El Risco del Hombre (Corteconcepción) y Malatao (Cartaya) (Sánchez Gullón et al., 2010), pero son los eucaliptos del azúcar de Sierra Cabello los de más antigua introducción, datada por Martín Bolaños en 1924, con presencia de ejemplares que destacan por su porte bien conformado,

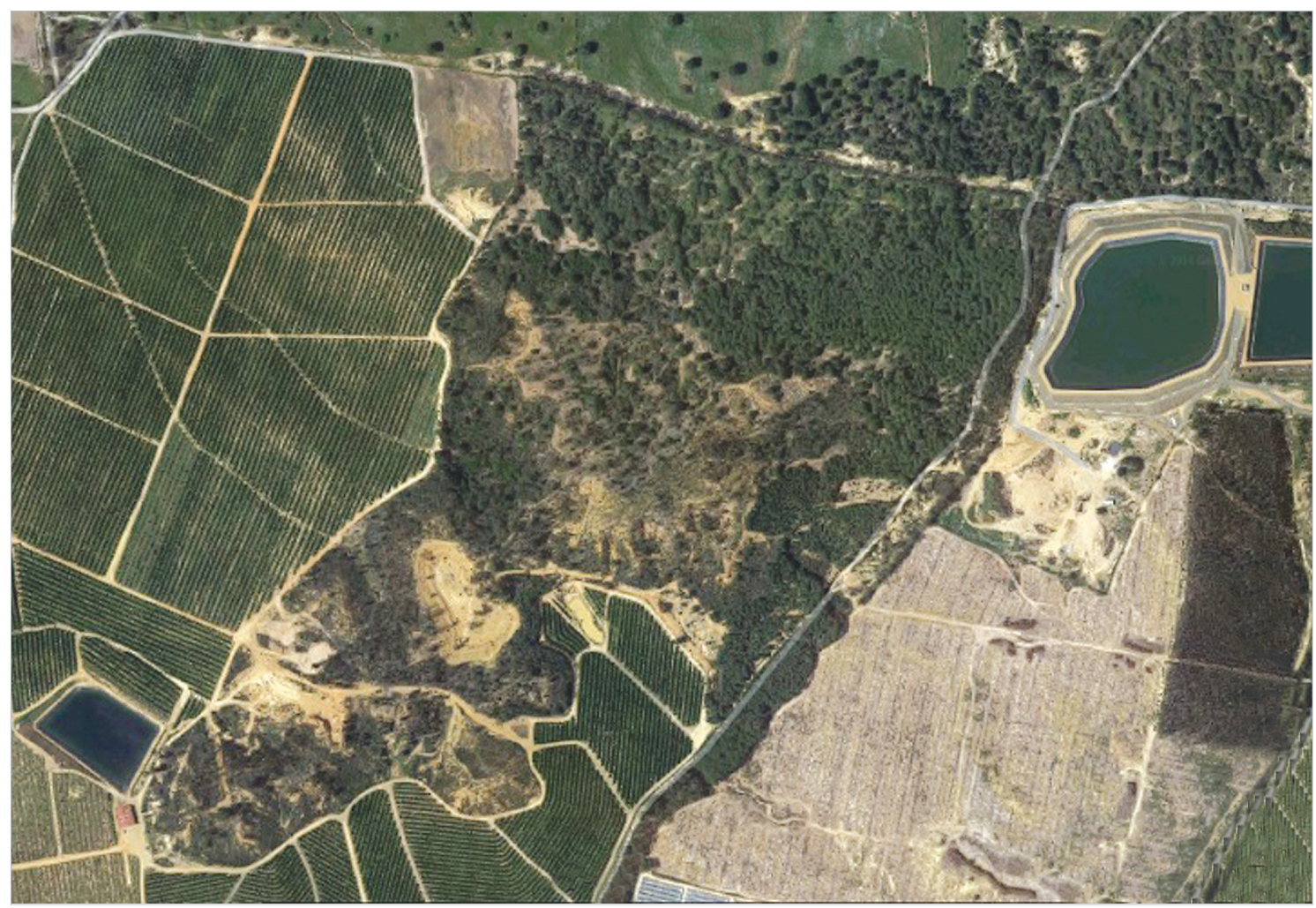

Figura 3. Fotografía aérea actual de las mesetas de Sierra Cabello y su entorno, en la que quedan en evidencia las extracciones de áridos realizadas y la transformación del eucaliptal en plantaciones de naranjo. 


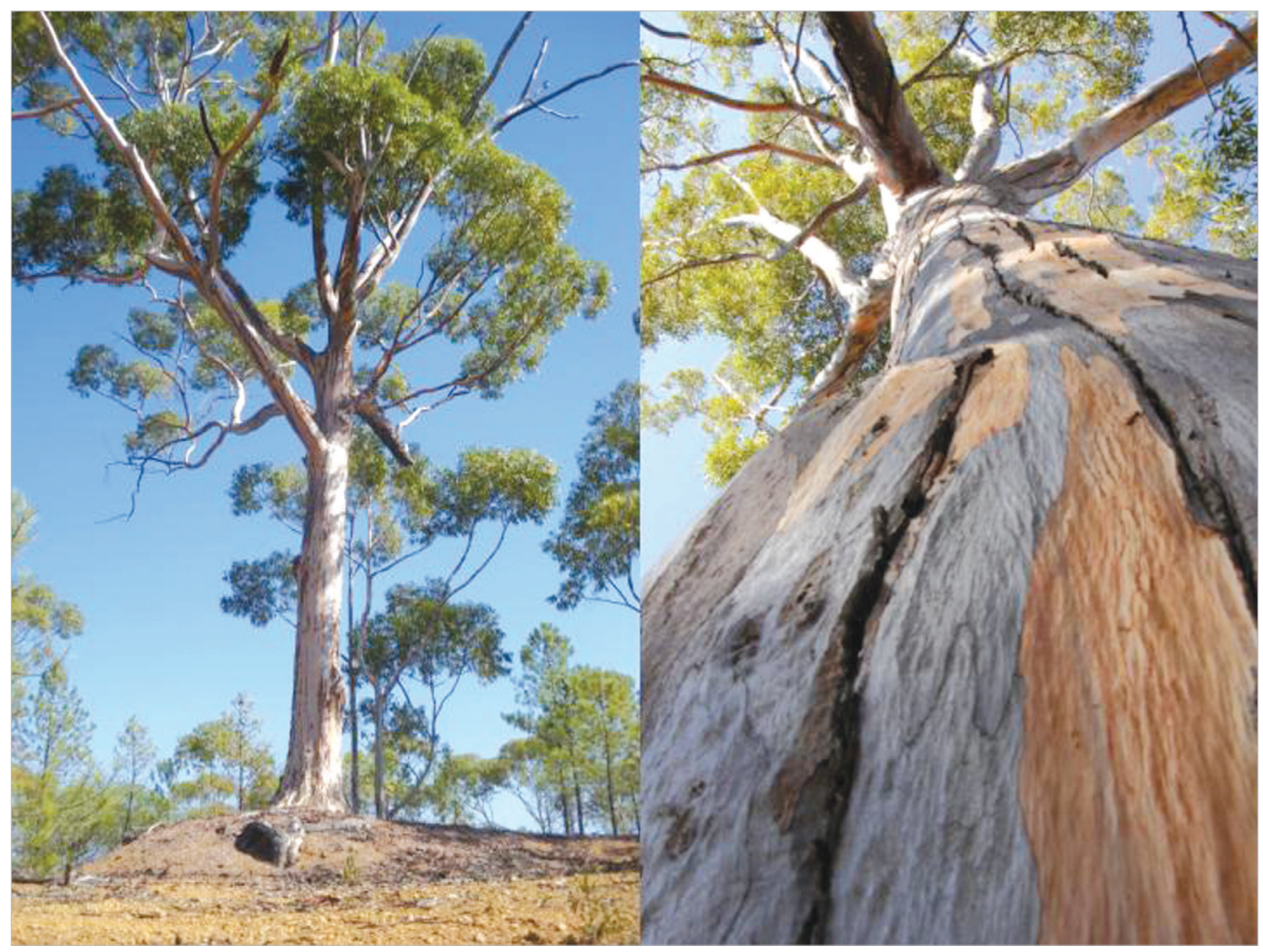

Figura 4. Ejemplares singulares de Eucalyptus cladocalyx del arboreto de Sierra Cabello.

color y brillo de sus cortezas, con tallas que se aproximan a la máxima descrita para la especie, gruesos diámetros de fuste y de ramas que conforman amplias copas aparasoladas, características que en su conjunto resultan muy llamativas en la zona y parecen indicar que la especie vegeta en una estación muy adecuada para su desarrollo (Fig. 4, tabla 4).

Tabla 4. Datos dendrométricos de un árbol tipo de Eucalyptus cladocalyx del arboreto de Sierra Cabello y comparación con los ofrecidos por la bibliografía española.

\begin{tabular}{lcccc}
\hline & $\begin{array}{c}\text { Árbol tipo } \\
\text { Sierra Cabello }\end{array}$ & $\begin{array}{c}\text { Martín Bolaños, } \\
\mathbf{1 9 5 5}\end{array}$ & $\begin{array}{c}\text { De la Lama } \\
\text { Gutiérrez, 1976 }\end{array}$ & $\begin{array}{c}\text { Ruiz de la Torre, } \\
\mathbf{2 0 0 6}\end{array}$ \\
\hline Porte & Arbóreo & Arbusto o árbol & Arbusto o árbol & $\begin{array}{c}\text { Mata arbustiva, } \\
\text { arbusto o árbol }\end{array}$ \\
\cline { 1 - 2 }$(\mathrm{m})$ & 23 & hasta $30-40$ & 9 a 40 & 6 a 30 \\
Tiámetro $(\mathrm{cm})$ & 89.13 & hasta 100 a 150 & hasta 150 & -- \\
\hline Perímetro a 1,30m $(\mathrm{cm})$ & 280 & -- & -- & -- \\
Perímetro en la base $(\mathrm{cm})$ & 560 & -- & -- & - \\
Diámetro de copa $(\mathrm{m})$ & N-S 19.60 & -- & -- & - \\
& E-O 18.10 & & & \\
\hline
\end{tabular}


Tabla 5. Distribución provincial y local de los eucaliptos catalogados como árboles singulares en Andalucía.

\begin{tabular}{|c|c|}
\hline Provincia & $\begin{array}{c}\text { Especie } \\
\text { Denominación (Término municipal) }\end{array}$ \\
\hline Almería & -- \\
\hline \multirow[t]{2}{*}{ Cádiz } & Eucalyptus camaldulensis \\
\hline & Eucalipto del Pinar de la Algaida (Sanlúcar de Barrameda) \\
\hline \multirow[t]{4}{*}{ Córdoba } & Eucalyptus camaldulensis \\
\hline & Eucalipto de la Fuente Agria de El Hoyo (Bélmez) \\
\hline & Eucalipto de la Hacienda de Nublos (Hornachuelos) \\
\hline & Eucaliptos de Las Grúas (E1 Carpio): alineación de cuatro eucaliptos \\
\hline Granada & -- \\
\hline \multirow[t]{3}{*}{ Huelva } & Eucalyptus camaldulensis \\
\hline & Eucalipto de La Cruz (Almonte) \\
\hline & Eucalipto de la Venta del Palacio de Doñana (Almonte) \\
\hline \multirow[t]{3}{*}{ Jaén } & Eucalyptus camaldulensis \\
\hline & Eucalipto del Arroyo de los Ríos (Guarromán) \\
\hline & Eucalipto del Arroyo del Torrito (Linares) \\
\hline Málaga & -- \\
\hline \multirow[t]{7}{*}{ Sevilla } & Eucalyptus camaldulensis \\
\hline & Eucalipto de la Hacienda Torrijos (Valencina de la Concepción) \\
\hline & Eucalipto de la Huerta de Malagón (Villanueva del Río y Minas) \\
\hline & Eucalipto de la Pizana (Gerena) \\
\hline & Eucalipto de Torre del Abad (Alcalá de Guadaira) \\
\hline & Eucalyptus citriodora \\
\hline & $\begin{array}{c}\text { Eucaliptos de la Casa de El Colmenar (Aznalcázar): alineación de cuatro } \\
\text { eucaliptos frente a la Casa Forestal de El Colmenar }\end{array}$ \\
\hline
\end{tabular}

Fuente: Sánchez Lancha (Dir. Fac.), 2003a-e, 2004a y b, 2010

En 6 de las 8 provincias andaluzas, todas menos Almería y Málaga, hay eucaliptos o pequeñas alineaciones catalogados como singulares (Tab. 5). Se trata generalmente de E. camaldulensis de grandes dimensiones, a excepción de los "Eucaliptos de la Casa de El Colmenar" (Aznalcázar, Sevilla) de la especie E. citriodora. Son árboles añosos, testimonio de la llegada de este género a Andalucía mediado el siglo XIX, cuando comenzaron las primeras experiencias con E. globulus, coetáneas con las realizadas en Galicia, Valencia, Cataluña, Asturias, Cantabria y País Vasco. Esta medida de conservación de la memoria forestal española se muestra incompleta al carecer los arboretos de eucaliptos de una protección especial, que podría materializarse en el caso de Andalucía en su catalogación como arboledas singulares, destacando aquellos ejemplares de mayor interés. 


\section{Conclusiones}

- Sierra Cabello, ubicada en un enclave biogeográfico particular, constituye un ejemplo único de las primeras plantaciones de eucaliptos realizadas en la provincia de Huelva, de la, que gracias a los trabajos de Martín Bolaños, existe una buena documentación botánica y selvícola.

- Los ejemplares singulares de eucalipto del azúcar Eucalyptus cladocalyx F. Muell. por su rareza, belleza y características dendrométricas merecen gozar de un estatus de protección especial.

- Por estas razones se propone la inclusión de Sierra Cabello y sus notables ejemplares de E. cladocalyx en el Inventario de Árboles y Arboledas Singulares de Andalucía.

\section{Agradecimientos}

A Paloma Gil Borrell (ETSIM-UPM), Carmen de Arana Moncada y José Manuel Grau Corbí (CIFOR-INIA) por su ayuda en la localización de la documentación inédita de Manuel Martín Bolaños, y a Enrique Sánchez Gullón por su colaboración en el inicio del trabajo de campo.

\section{Bibliografía}

Boletín Oficial de la Junta de Andalucía, 2003. LEY 8/2003, de 28 de octubre, de la flora y la fauna silvestres. BOJA núm 218, 12/11/2003.

Boletín Oficial de la Junta de Andalucía, 2012. Decreto 23/2012, de 14 de febrero, por el que se regula la conservación y el uso sostenible de la flora y la fauna silvestres y sus hábitats. BOJA núm 60, 27/03/2012.

Butler Sierra, I., 2016. Los trabajos de Manuel Martín Bolaños sobre la vegetación y la flora forestal de la provincia de Huelva. Aplicación al análisis de cambios espaciotemporales en el Paraje Natural Sierra Pelada y Rivera del Aserrador. Tesis Doctoral. Universidad de Huelva.

De la Lama Gutiérrez, G., 1976. Atlas del eucalipto. Tomo I. Información y ecología. Ministerio de Agricultura, Instituto Nacional de Investigaciones Agrarias, Instituto para la Conservación de la Naturaleza, Sevilla.

Instituto Geológico y Minero de España, 1983. Mapa Geológico de España E. 1:50.000. Hoja 981.Gibraleón. Segunda Serie. Servicio de Publicaciones, Ministerio de Industria y Energía, Madrid.

Martín Bolaños, M., 1928. Flora y Mapa Forestal. Los eucaliptos de Sierra Cabello. Instituto Forestal de Investigaciones y Experiencias Agronómicas y Forestales, año I $\mathrm{n}^{\mathrm{o}} 1,5$ 15.

Martín Bolaños, M., 1946. Impresiones comentadas sobre los eucaliptos de Sierra Cabello. Ministerio de Agricultura, Dirección General de Montes, Caza y Pesca Fluvial, Instituto 
Forestal de Investigaciones y Experiencias, año XVII núm. 32, Madrid.

Martín Bolaños, M., 1955. Eucaliptos de mayor interés para España. Ministerio de Agricultura, Dirección General de Montes, Caza y Pesca Fluvial, Instituto Forestal de Investigaciones y Experiencias, año XXVI núm. 73, Madrid.

Rivas Martínez, S., 2007. Mapa de series, geoseries y geopermaseries de vegetación de España [Memoria del Mapa de Vegetación potencial de España]. Parte I. Itinera Geobotanica (Nueva Serie). Vol. 17. 435 pp.

Ruiz de la Torre J., 2006. Flora mayor. Ministerio de Medio Ambiente, Organismo Autónomo de Parques Nacionales, Madrid.

Sánchez Gullón, E., Caraballo Martínez, J.M., Ruiz, F., 2010. Una visión histórica de los arboretos de eucaliptos de Huelva. Boletín del CIDEU 8-9: 43-56.

Sánchez Lancha, A. (Dir. Fac.),

- 2003a. Árboles y Arboledas Singulares de Andalucía. Cádiz. Junta de Andalucía, Consejería de Medio Ambiente, Sevilla.

- 2003b. Árboles y Arboledas Singulares de Andalucía. Córdoba. Junta de Andalucía, Consejería de Medio Ambiente, Sevilla.

- 2003c. Árboles y Arboledas Singulares de Andalucía. Granada. Junta de Andalucía, Consejería de Medio Ambiente, Sevilla.

- 2003d. Árboles y Arboledas Singulares de Andalucía. Huelva. Junta de Andalucía, Consejería de Medio Ambiente, Sevilla.

- 2003e. Árboles y Arboledas Singulares de Andalucía. Sevilla. Junta de Andalucía, Consejería de Medio Ambiente, Sevilla.

- 2004a. Árboles y Arboledas Singulares de Andalucía. Jaén. Junta de Andalucía, Consejería de Medio Ambiente, Sevilla.

- 2004b. Árboles y Arboledas Singulares de Andalucía. Málaga. Junta de Andalucía, Consejería de Medio Ambiente, Sevilla.

- 2010. Árboles y Arboledas Singulares de Andalucía. Almería. Junta de Andalucía, Consejería de Medio Ambiente, Sevilla. 
